\title{
A Rare Case of Gastric Schwannoma: A Case Report and Literature Review
}

\author{
Senichiro Yanagawa ${ }^{a}$ Kenichi Kagemoto ${ }^{b}$ Hidehiro Tanji ${ }^{a}$ \\ Shinya Kodama ${ }^{a}$ Yukio Takeshima ${ }^{c}$ Kazuo Sumimoto ${ }^{a} d$ \\ aDepartment of Surgery, Yoshida General Hospital, Akitakata City, Japan; bepartment \\ of Internal Medicine, Yoshida General Hospital, Akitakata City, Japan; 'Department of \\ Pathology, Graduate School of Biomedical and Health Sciences, Hiroshima University, \\ Hiroshima, Japan; dDepartment of Gastroenterological and Transplant Surgery, Graduate \\ School of Biomedical and Health Sciences, Hiroshima University, Hiroshima, Japan
}

\section{Keywords}

Gastric schwannoma · Surgical resection · Laparoscopic surgery

\begin{abstract}
In general, schwannoma is a benign and slow-glowing neoplasm that rarely occurs in the gastrointestinal tract as a submucosal tumor (SMT), with the most common site being the stomach. As gastric schwannoma (GS) is a rare tumor, there is limited data in the literature about its clinical features. The diagnosis of schwannoma can only be made by pathological examination with positive staining for S-100 protein. It is necessary to obtain an accurate diagnosis to introduce optimal treatment options preoperatively. However, a precise diagnosis of GS is difficult, even with modern imaging techniques. On the other hand, a complete resection with a negative surgical margin (R0) of GS is considered the best treatment, with an excellent prognosis. We present a case of a 66 -year-old female patient who underwent laparoscopic-assisted wedge gastrectomy for gastric SMT, pathologically diagnosed as GS, with positive staining for S-100 protein and negative for c-kit and CD34. At 12-month follow-up after surgery, there was no recurrence or metastasis of GS. Our treatment is appropriate and effective in case of GS exceeding $50 \mathrm{~mm}$.

\section{Introduction}

In general, schwannoma is a benign neurogenic tumor originating from Schwann cells. It is slow growing and enfolds the axons of the peripheral nerves. Theoretically, schwannoma can develop anywhere along the peripheral course of the nerve. However, it commonly occurs 
in the head or neck, and rarely in the gastrointestinal tract, where the most common site is the stomach.

Gastric schwannoma (GS) accounts for less than 1\% of all gastric tumors [1, 2]. GS arises from the nerve sheath of Auerbach's or Meissner's plexus and is observed endoscopically as a submucosal tumor (SMT) [3, 4]. Although GS is a benign neoplasm with an excellent prognosis after complete surgical resection, the clinical or imaging features have not been sufficiently specific to enable a precise preoperative diagnosis, because gastric SMTs are similar in their gross and imaging findings, and the diagnosis by biopsy does not have a high accuracy rate. At present, only a pathological examination can distinguish a schwannoma from other SMTs, with positive staining for S-100 protein, and negative for c-kit, CD34, desmin, or smooth muscle actin $[1,4,5]$.

Herein, we present a case of GS located at the greater curvature of the stomach. The surgical procedure we chose is considered simple and appropriate with several perspectives in case of GS exceeding $50 \mathrm{~mm}$.

\section{Case Report}

A 66-year-old female had a history of laparoscopic cholecystectomy. She visited our hospital due to an abnormal upper gastrointestinal X-ray at the medical checkup (Fig. 1a). An upper endoscopy revealed an elevated submucosal gastric mass on the greater curvature, and a normal overlying mucosa without ulceration (Fig. 1b). A contrast-enhanced computed tomography (CT) showed the tumor measuring $52 \mathrm{~mm}$ on the axial section (Fig. 1c), with extramural growth on the coronal section in the stomach (Fig. 1d). The serum carcinoembryonic antigen level was within the normal range. Laparoscopic-assisted wedge gastrectomy was performed and a complete resection with negative surgical margin (R0) was obtained (Fig. 2a, b). Pathological examination with hematoxylin and eosin staining of the specimen revealed a solid tumor which was highly cellular and consisted of spindle cells (Fig. 3a), surrounded by a peripheral lymphoid cuff (Fig. 3b). Mitosis was scattered, with $0-5$ mitoses per 50 high-power field. Immunohistochemistry revealed that the tumor cell was strongly positive for S-100 protein (Fig. 3c) and negative for CD34 and c-kit (Fig. 3d, e). In addition, the MIB-1 max labeling index was $5 \%$ (Fig. 3f). These pathological results had confirmed the diagnosis as GS.

She displayed no complications and was discharged on day 8 after surgery. At the 12-month follow-up after surgery, there has been no recurrence or metastasis of GS on CT and upper endoscopy.

\section{Discussion and Conclusion}

Schwannoma is generally a slow-growing and benign neoplasm that rarely occurs in the gastrointestinal tract. Gastrointestinal schwannoma was first reported by Daimaru et al. [1], who identified schwannoma as a primary gastrointestinal tumor based on the positive S-100 protein immunostaining. GS, which is the most common gastrointestinal schwannoma, accounts for only $0.2 \%$ of all gastric tumors and, principally, involves the submucosa and muscularis propria $[1,2]$. Although GS is mostly benign, the differential diagnosis is gastrointestinal stromal tumor (GIST). On account of the typical presentation as SMT with spindle cell histology, GS and GIST are grossly similar. In the diagnosis of such an SMT, an endoscopic biopsy can lead to false-negative results and is thus ineffective. On the other hand, endoscopic ultrasound-fine needle aspiration (EUS-FNA) could possibly aid in the diagnosis [4, 6-8]. 

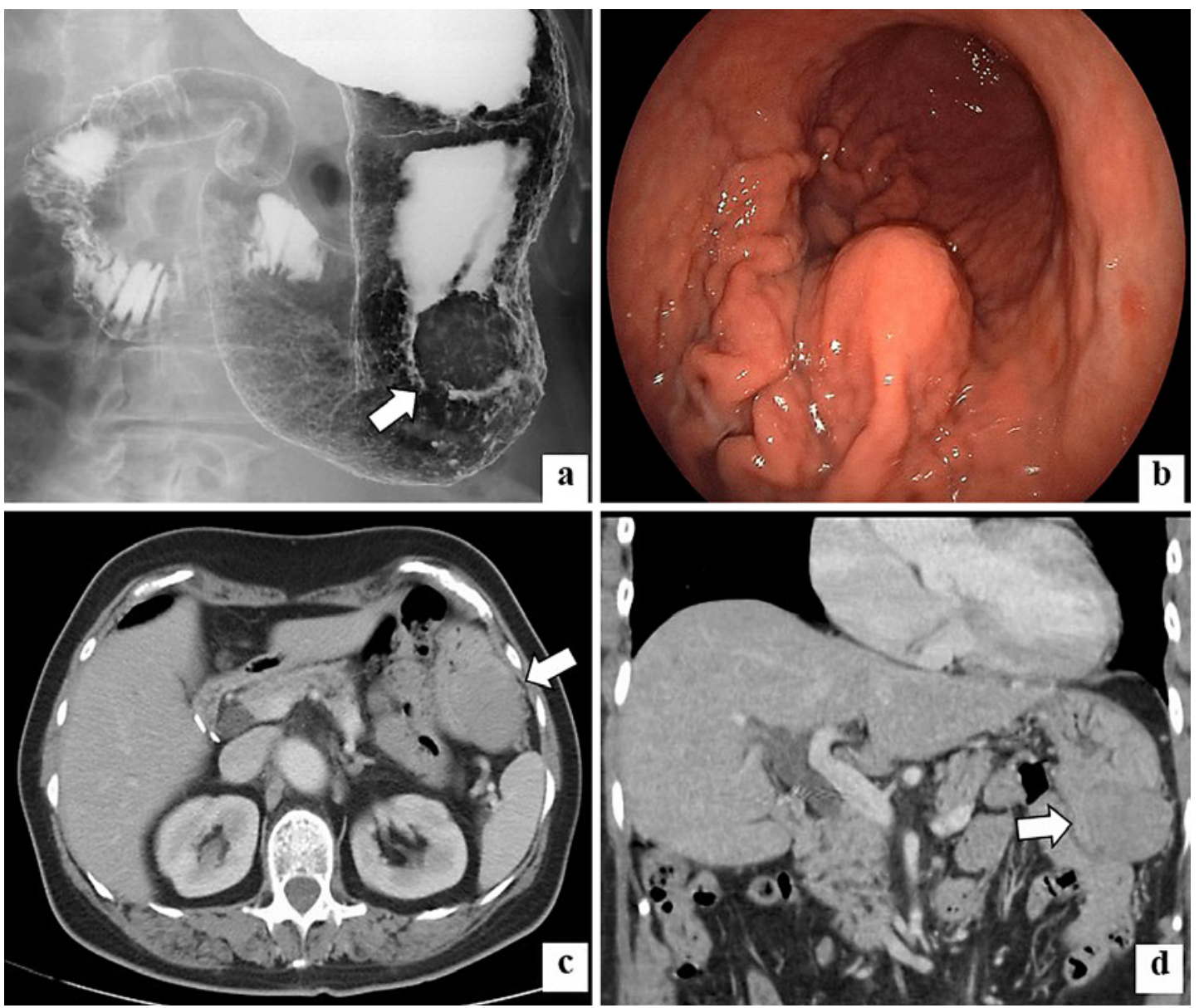

Fig. 1. Preoperative imaging inspection findings. a The shadow defect with bridging fold, about $40 \mathrm{~mm}$ in diameter, was pointed in the middle of the gastric body in the upper gastrointestinal X-ray. b Upper endoscopy revealed an elevated submucosal mass, about $40 \mathrm{~mm}$ in diameter, in the gastric body at the greater curvature. c A contrast-enhanced CT in the axial section showed the tumor measuring $52 \mathrm{~mm}$ in the stomach. d A contrast-enhanced CT in the coronal section showed the tumor with extramural growth in the stomach.
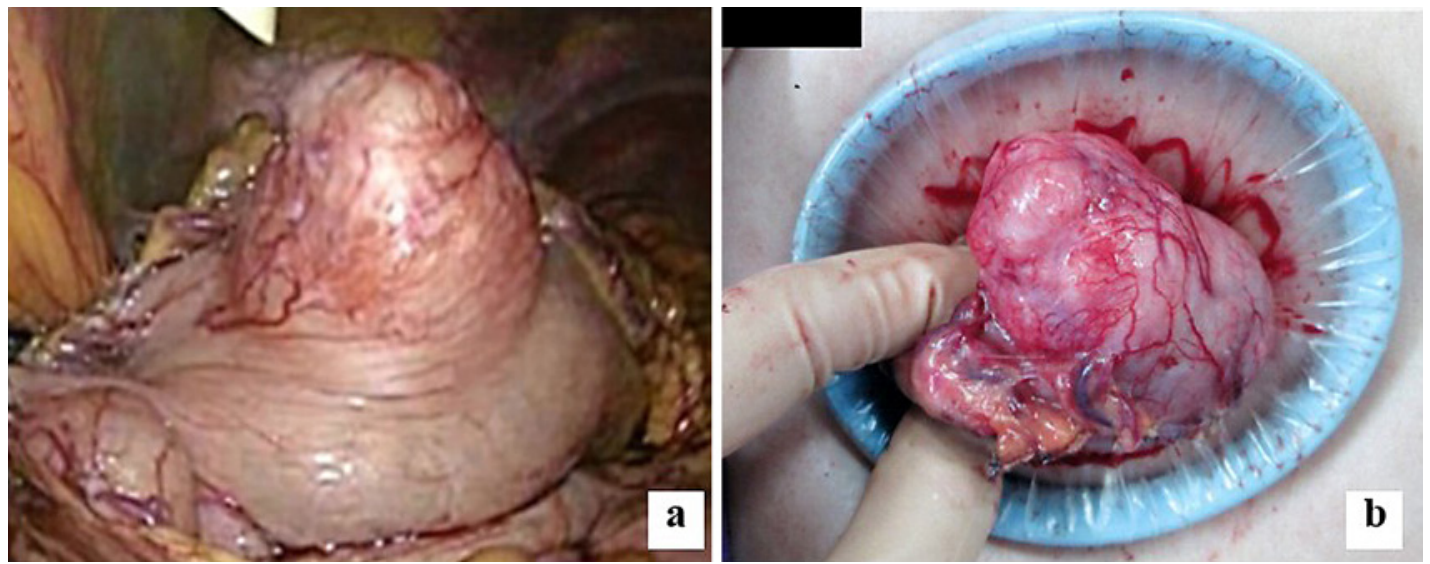

Fig. 2. Intraoperative findings. a The great omentum was dissected by laparoscopic surgery to expose the tumor. The tumor was then pulled just below the port of the laparoscope. $\mathbf{b}$ The part of the stomach with the tumor was removed from the body. Wedge gastrectomy was performed. 
Fig. 3. The results of HE staining and immunostaining. a The tumor shows the lesion, composed of spindle cells with nuclei, arranged in a palisade. b There is lymphocytic cuffing at the peripheral part of the tumor. c The tumor cells are positive for S-100 protein. d The tumor cells are negative for CD34. e The tumor cells are negative for the c-kit. $\mathbf{f}$ The MIB-1 labeling index is $5 \%$.

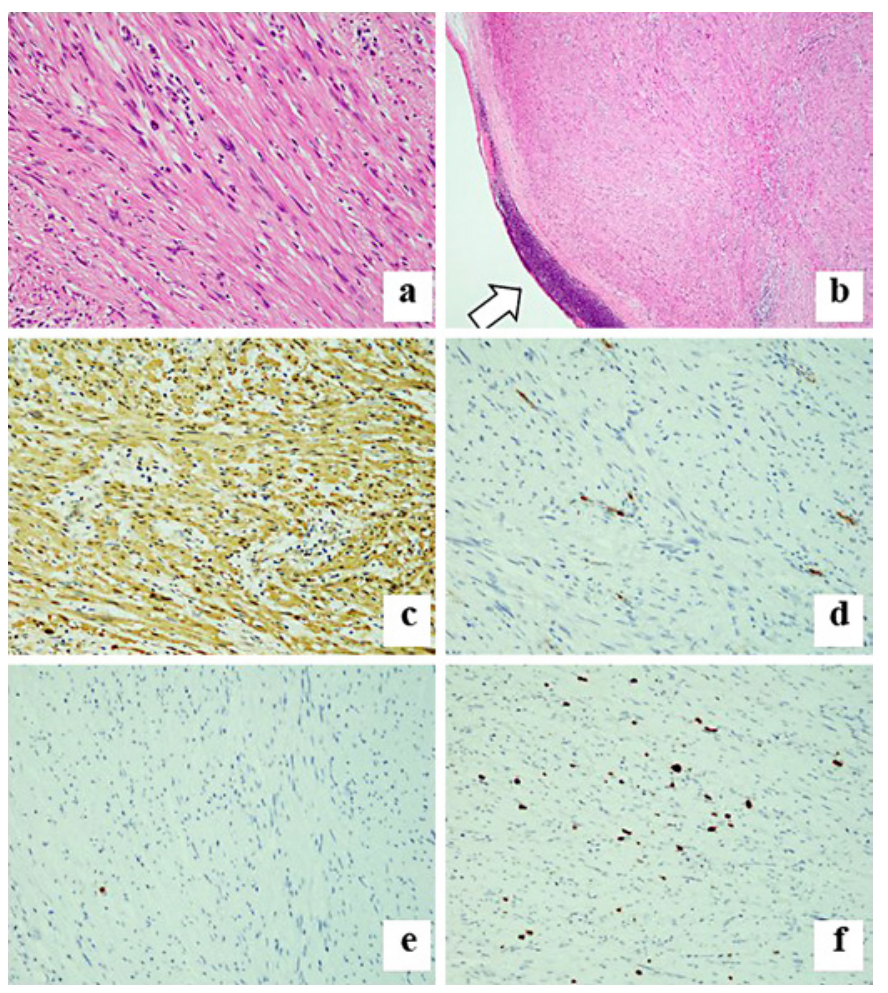

However, there is no consensus on the accuracy rate; additionally, routine EUS-FNA is not recommended for primary resectable GIST by The National Comprehensive Cancer Network (NCCN) guidelines, due to the risk of tumor rupture and spread, which is associated with poorer prognosis [4, 6-9].

The precise preoperative diagnosis to distinguish between GS and another SMT remains difficult, even with modern imaging modalities such as CT or ${ }^{18} \mathrm{~F}$-fluorodeoxyglucose-positron emission tomography (FDG-PET) $[2,4,10]$. For example, GS displays as round and the pattern of contrast enhancement is classified as either homogeneous or heterogeneous on CT; furthermore, FDG-PET does not help in evaluating whether tumors are benign or malignant $[2,4]$. Thus, none of these modalities has shown the diagnostic features specific to GS. However, a complete resection with a negative surgical margin is the curative treatment for GS, as with GIST or other SMTs [3-5, 7, 9]. Recently, laparoscopic and endoscopic cooperative surgery (LECS) has been increasingly applied for gastric SMTs [11]. The conventional LECS procedure runs the potential risk of gastric content or tumor cell spilling into the abdominal cavity. Kikuchi et al. [12] developed a modified LECS procedure named "closed LECS" to avoid this problem. They concluded that "closed LECS" is indicated for gastric SMTs, $30 \mathrm{~mm}$ or less in diameter. In addition, LECS requires highly sophisticated laparoscopic and endoscopic techniques or equipment with high medical costs. Therefore, in the treatment of such SMTs, any approach in which complete surgical resection is performed safely would be acceptable.

The possibility of malignancy could not be ruled out in our case, because a case of malignant tumor exceeding $50 \mathrm{~mm}$ in diameter has been reported [13]. In order to prevent its rupture, the gastric tumor was removed from the abdominal cavity under laparoscopic guidance. A wedge gastrectomy was performed, with the tumor removed out of the body. We did not carry out a preoperative diagnosis with useful modern examinations, such as EUS-FNA or FDG-PET, and did not employ the latest surgical methods, including LECS. However, in view of medical costs, minimal invasiveness and safety of the patient, and oncological aspects, the 
treatment we chose is beneficial enough for GS exceeding $50 \mathrm{~mm}$, and there is no disadvantage to the patient.

GS is a benign neoplasm with no recurrence irrespective of its size [14]. Nevertheless, we believe that regular follow-up is necessary for GS because of the report of such a malignant case.

\section{Acknowledgements}

The authors would like to thank the patient for allowing us to report her clinical information and data. We would like to thank Editage (www.editage.com) for English language editing.

\section{Statement of Ethics}

Written informed consent was obtained from the patient.

\section{Disclosure Statement}

The authors have no conflicts of interest to declare.

\section{Funding Sources}

The authors received no financial support for the research, authorship, or publication of this article.

\section{Author Contributions}

S.Y. made a substantial contribution toward the concept and design of the study and in data acquisition and interpretation. K.K., H.T., S.K., Y.T., and K.S. were involved in drafting the manuscript and critical revision for intellectual content. S.Y. approved the final version of the manuscript submitted for publication. All authors read and approved the final manuscript.

\section{References}

1 Daimaru Y, Kido H, Hashimoto H, Enjoji M. Benign schwannoma of the gastrointestinal tract: a clinicopathologic and immunohistochemical study. Hum Pathol. 1988 Mar;19(3):257-64.

2 Hong HS, Ha HK, Won HJ, Byun JH, Shin YM, Kim AY, et al. Gastric schwannomas: radiological features with endoscopic and pathological correlation. Clin Radiol. 2008 May;63(5):536-42.

3 Fujiwara S, Nakajima K, Nishida T, Takahashi T, Kurokawa Y, Yamasaki M, et al. Gastric schwannomas revisited: has precise preoperative diagnosis become feasible? Gastric Cancer. 2013 Jul;16(3):318-23.

4 Tao K, Chang W, Zhao E, Deng R, Gao J, Cai K, et al. Clinicopathologic features of gastric schwannoma: 8-year experience at a single institution in China. Medicine (Baltimore). 2015 Nov; 94(45):e1970.

5 Mekras A, Krenn V, Perakis A, Croner RS, Kalles V, Atamer C, et al. Gastrointestinal schwannomas: a rare but important differential diagnosis of mesenchymal tumors of gastrointestinal tract. BMC Surg. 2018 Jul 25; 18(1):47.

6 Mekky MA, Yamao K, Sawaki A, Mizuno N, Hara K, Nafeh MA, et al. Diagnostic utility of EUS-guided FNA in patients with gastric submucosal tumors. Gastrointest Endosc. 2010 May;71(6):913-9. 
7 Hu J, Liu X, Ge N, Wang S, Guo J, Wang G, et al. Role of endoscopic ultrasound and endoscopic resection for the treatment of gastric schwannoma. Medicine (Baltimore). 2017 Jun;96(25):e7175.

8 Nishida T, Blay JY, Hirota S, Kitagawa Y, Kang YK. The standard diagnosis, treatment, and follow-up of gastrointestinal stromal tumors based on guidelines. Gastric Cancer. 2016 Jan;19(1):3-14. Epub 2015 Aug 15.

9 Demetri GD, Benjamin RS, Blanke CD, Blay JY, Casali P, Choi H, et al. NCCN Task Force report: management of patients with gastrointestinal stromal tumor (GIST)-update of the NCCN clinical practice guidelines. J Natl Compr Canc Netw. 2007 Jul;5(Suppl 2):S1-29.

10 Wang W, Cao K, Han Y, Zhu X, Ding J, Peng W. Computed tomographic characteristics of gastric schwannoma. J Int Med Res. 2019 May;47(5):1975-86.

11 Niimi K, Ishibashi R, Mitsui T, Aikou S, Kodashima S, Yamashita H, et al. Laparoscopic and endoscopic cooperative surgery for gastrointestinal tumor. Ann Transl Med. 2017 Apr;5(8):187.

12 Kikuchi S, Nishizaki M, Kuroda S, Tanabe S, Noma K, Kagawa S, et al. Nonexposure laparoscopic and endoscopic cooperative surgery (closed laparoscopic and endoscopic cooperative surgery) for gastric submucosal tumor. Gastric Cancer. 2017 May;20(3):553-7.

13 Takemura M, Yoshida K, Takii M, Sakurai K, Kanazawa A. Gastric malignant schwannoma presenting with upper gastrointestinal bleeding: a case report. J Med Case Rep. 2012 Jan 25;6:37.

14 Hong X, Wu W, Wang M, Liao Q, Zhao Y. Benign gastric schwannoma: how long should we follow up to monitor the recurrence? A case report and comprehensive review of literature of 137 cases. Int Surg. 2015 Apr; 100(4): 744-7. 